\title{
Methods and instrumentation for undergraduate teaching: conduction of biopotentials in the earthworm.
}

\author{
Lucas Y. Tanio (IC), José W. M. Bassani (PQ)
}

\begin{abstract}
In this work, we present a preparation with earthworms (Eisenia fetida; Savigny, 1826) and instrumentation for teaching basic neurophysiology on Biomedical Engineering laboratory classes.
\end{abstract}

Key words: Action potential, earthworm, conduction velocity.

\section{Introduction}

Studying neurobiology and physiology requires a good teacher-student interaction. Some models and simulations can be of a good help using simple experiments. In this work, we present a standard protocol and instrumentation to be used in physiology and biomedical engineering laboratory classes, using earthworms (Eisenia fetida; Savigny, 1826). The protocol should be used as a tool for helping understanding some basic concepts of nerve action potentials (AP) triggering and conduction. Here we present results of experiments performed for measuring conduction velocity in nerve of anesthetized animals.

\section{Results and Discussion}

According to the Brazilian Law, experiments with invertebrates do not require an ethical committee approval. Care was taken, however, to avoid any inappropriate use of the animals and to work with a minimum number of individuals. Earthworms were anesthetized in an alcohol bath $(15 \%$ ethanol, 5 - $7 \mathrm{~min}$ ). After that, the earthworms were washed in tap water to remove the alcohol solution'. Animals were pinned on a silicon chamber with a printed ruler in the bottom by three stainless steel electrodes separated by about $20 \mathrm{~mm}$. For measuring AP conduction in LGF the signal electrodes were placed close to the earthworm hind end and the reference in the opposite side (front end). When MGF was accessed the signal electrodes were placed close to the Clittellum and the common electrode was pinned $0.02 \mathrm{~m}$ toward the hind end of the animal. The circuit consisted of a two gain stage amplifier and a band-pass filter (based on the Backyard Brains Inc., Ann Arbor, MI, USA circuit) ${ }^{1}$. The earthworms were mechanically stimulated with a non-conductive rod in the front end to observe the response of the medial giant nerve fibers (MGF) and on the hind end to access the lateral giant nerve fibers (LGF). Conduction velocity was calculated from the distance between the electrodes and time difference between electrical spikes (Figure 1). Data were expressed as mean \pm SEM. Groups were compared by Student's t test for unpaired samples. Figure 1 shows a typical recording from LGF. The mean conduction velocities were $17.7 \pm 2.7 \mathrm{~m} / \mathrm{s}$ and $8,7 \pm 1,3 \mathrm{~m} / \mathrm{s}$ in MGF and LGF, respectively. As predicted from the literature action potentials travel faster in MGF than in LGF $(p<0.05, N=3)$.

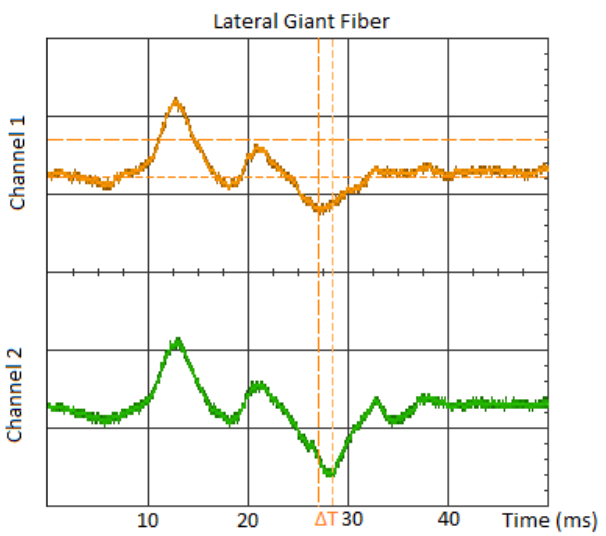

Figure 1. Recordings from the earthworm LGF. Channel 1 shows signal at the electrode closer to earthworm hind end. Channel 2 shows the same signal on a second electrode. Distance between electrodes was $0.011 \mathrm{~m}$. The vertical dashed lines show time difference $(\Delta T=0.0014 \mathrm{~s})$ between the signal spikes.

\section{Conclusions}

Preparation must be improved to make experiments applicable in laboratory classes.

\section{Acknowledgement}

CEB-UNICAMP staff for technical assistance and $\mathrm{CNPq}$ for the financial support.

${ }^{1}$ Shannon, K.M.; Gage, G.J.; Jankovic, A.; Wilson, W.J.; Marzullo, T.C. Portable conduction velocity experiments using earthworms for the college and high school neuroscience teaching laboratory, Adv physiol Educ, 38 (1): 62-70, 2014. 